\title{
A NOTE ON DIVIDED POWERS IN A HOPF ALGEBRA
}

\author{
EDWARD HALPERN
}

The algebraic structure of a Hopf algebra over a field was established by A. Borel [1] (see Théorème 6.1). The theorem asserts that if a Hopf algebra is finitely generated in each dimension and the field is perfect then it is algebraically isomorphic to a tensor product of monogenic exterior and polynomial algebras (the latter possibly truncated if the characteristic is prime). Under more restrictive assumptions a similar structure theorem is proved in [5] (see Theorem 7.13) in the case where the field is replaced by an integral domain. In this note we shall make use of the latter theorem in the case where the Hopf algebra has a system of divided powers and then apply the result to $H$-spaces.

1. Twisted and divided powers. Throughout, $J$ will denote a principal ideal domain. Let $H$ be a (unitary) $J$-module. An element $x \in H$ is divisible by $a \in J, a \neq 0$, if there is an element $y \in H$ such that $a y=x$; if $x$ is divisible only by units in $J$ we say $x$ is a divided element. A greatest divisor of $x$ is an element $g(x) \in J$ which divides $x$ and has the property: if $a \in J$ divides $x$ then $a$ divides $g(x)$. If $g(x)$ and $g^{\prime}(x)$ are greatest divisors of $x$, then

$$
g(x)=a g^{\prime}(x), \quad g^{\prime}(x)=a^{\prime} g(x), \quad a, a^{\prime} \in J^{6}
$$

from which it follows that $\left(a a^{\prime}-1\right) g(x)=0$. Since $J$ has no zero divisors, $a$ and $a^{\prime}$ are units. Thus $g(x)$ is unique to within unit factors.

Proposition 1.1. If $H$ is a free $J$-module then:

(a) Every nonzero $x$ may be represented

$$
x=g(x) \bar{x}
$$

where $g(x)$ is a greatest divisor, $\bar{x}$ a divided element, and (1.1) is unique to within unit factors.

(b) If $\bar{y}$ is a divided element and $a z=b \bar{y}$, then $a$ divides $b$.

Proof. (a) The uniqueness follows at once from the preceding discussion. Let $x \neq 0$; we may write $x=\sum a_{i} x_{i}, a_{i} \neq 0$, where the $x_{i}$ are elements of a basis for $H$. Factoring out the greatest common divisor of the $a_{i}$ we obtain a representation (1.1).

(b) It suffices to show that $b$ is a greatest divisor of $x=b \bar{y}$. Writing

Presented to the Society, February 28, 1959 under the title $A$ theorem on Hopf algebras with divided powers; received by the editors September 17, 1959. 
(1.1) for $x$, we see that $g(x)=b c$ for some $c$ in $J$. Then $b(c \bar{x}-\bar{y})=0$, from which $c \bar{x}=\bar{y}$ and hence $c$ is a unit. Thus $b$ is a greatest divisor of $x$.

Now assume $H$ is a $J$-algebra with a unit 1 . We recall that the height of a nonzero element $u$ is the least integer $h(u) \geqq 2$ such that $u^{h(u)}=0$. If no such integer exists we define $h(u)=\infty$. If $H$ is graded and anticommutative and $u$ is a homogeneous element not in the center of $H$, then $u$ must have odd degree and $J$ characteristic not 2 . It follows that $h(u)=2$.

By a sequence of twisted powers for $x \in H$ we shall mean a sequence of linearly independent divided elements $\left\{x^{[m]}, 0 \leqq m<h(x)\right\}$ such that

$$
g\left(x^{m}\right) x^{[m]}=x^{m},
$$

where the $g\left(x^{m}\right)$ are greatest divisors of $x^{m}$ such that

$$
g\left(x^{0}\right)=1, \quad g\left(x^{1}\right)=1 .
$$

Thus the $g\left(x^{m}\right)$ are nonzero elements and

$$
x^{[0]}=1, \quad x^{[1]}=x .
$$

Proposition 1.2. Let $H$ be a free module and graded J-algebra. If $x \in H^{i}(i>0)$ is a divided element then it possesses a sequence of twisted powers; any two such sequences for $x$ differ at most by unit factors. The submodule spanned by a sequence of twisted powers is a subalgebra with multiplication given by

$$
x^{[r]} x^{[s]}=\left\{\begin{array}{lr}
0, & r+s \geqq h(x), \\
t_{r, 8}(x) x^{[m]}, & r+s=m<h(x),
\end{array}\right.
$$

where

$$
t_{r, s}(x)=\frac{g\left(x^{m}\right)}{g\left(x^{r}\right) g\left(x^{s}\right)} .
$$

Proof. For each $m, 0 \leqq m<h(x)$, choose a representation (1.2) with (1.3) holding (any two such representations differ by unit factors). Since $H$ is graded it is clear that the $x^{[m]}$ are linearly independent. Now let $0 \leqq r<h(x), 0 \leqq s<h(x)$, and $r+s=m$; then

$$
x^{m}=x^{r} x^{s}=g\left(x^{r}\right) g\left(x^{s}\right) x^{[r]} x^{[s]} .
$$

If $m \geqq h(x)$ then $x^{m}=0$; hence, it follows that $x^{[r]} x^{[8]}=0$. Suppose $m<h(x)$. Comparing (1.7) with (1.2) we see that $g\left(x^{r}\right) g\left(x^{s}\right)$ must divide $g\left(x^{m}\right)$ and (1.5) follows.

The sequence $t(x)=\left\{t_{r, s}(x)\right\}$ is determined to within unit factors 
and will be called the type of $x$. The subalgebra is said to be pseudogenerated by $x$ and is clearly independent of the choice of twisted powers; we shall denote it by $J[x, h(x), t(x)]$. We make the following observations:

(i) If $t_{r, s}(x)$ is a unit for all $r+s<h(x)$ we say $x$ is of ordinary type. We may evidently choose $x^{[m]}=x^{m}$, and hence

$$
J[x, h(x), t(x)]=J[x] /\left(x^{h}\right), \quad h=h(x) .
$$

(ii) If $t_{r, s}(x)=a_{r, 8} \cdot(r, s)$ for $r+s<h(x)$, where the $a_{r, s}$ are units in $J$ and $(r, s)$ denotes the binomial coefficient $(r+s) ! / r ! s$ ! we shall say that $x$ is of binomial type. We may adjust the choices of the twisted powers so that $a_{r, 8}=1$. From (1.6) it then follows that we may rewrite (1.2) as

$$
m ! x^{[m]}=x^{m} \quad 0 \leqq m<h(x) .
$$

In analogy with (1.8) we shall write

$$
J[x, h(x), t(x)]=J\langle x\rangle /\left(x^{h}\right), \quad h=h(x) .
$$

(iii) More generally, if $t_{r, s}(x)$ divides $(r, s)$ for all $r+s<h(x)$ we shall say that $x$ is of pseudobinomial type.

REMARKs. If $h(x)=2$ then $J[x, h(x), t(x)]$ is the monogenic exterior algebra $\Lambda_{J}(x)$ and, moreover, is both of ordinary and binomial type.

Let $J$ have prime characteristic $p$. If $x$ is of binomial type then $(1.2)^{\prime}$ implies that $h(x) \leqq p$. If $x$ is of pseudobinomial type and $h(x)=p$ then $x$ is of both ordinary and binomial type (this follows from the fact that $(r, s)$ is a unit if $r+s<p)$.

Let $H$ be a $J$-module. If $X$ is a subset of $H$, by its rational closure $|X|$ we shall mean the set of all $y \in H$ such that $a y \in X$ for some nonzero $a \in J$. Clearly, $X \subset|X|$, and $X \subset X^{\prime}$ implies $|X| \subset\left|X^{\prime}\right|$. Moreover, if $X$ is a submodule then so is $|X|$. The following general lemma is readily proved:

Lemma. Let $H$ be a free finitely generated $J$-module. A submodule $G \subset H$ is a direct summand if and only if $G=|G|$.

Let $H$ be an $J$-algebra. Let $P(X)$ denote the subalgebra generated by the elements of $X$; then $|P(X)|$ is a subalgebra of $H$. If $H=|P(X)|$ we call $X$ a system of pseudogenerators for $H$; it is a minimal system if

$$
x \in|P(X-\{x\})| \quad \text { for all } x \in X .
$$

If the inclusion $i: X \rightarrow H$ induces an algebra monomorphism

$$
\bigotimes_{x \in X} J[x] /\left(x^{h(x)}\right) \rightarrow H
$$


we say that $X$ is a special system. If $H$ is graded, the elements of $X$ are required to be homogeneous. (Note that over a field the concepts of generating and pseudogenerating systems coincide.)

A graded module is said to be of finite type if each homogeneous submodule is finitely generated.

Proposition 1.3. If $H$ is a torsion-free graded J-algebra of finite type then $J[x, h(x), t(x)]$ is a direct summand.

Proof. Let $u$ be a nonzero element of $|J[x, h(x), t(x)]|$. Then there is an element $a \in J, a \neq 0$, such that

$$
a u=\sum a_{m} x^{[m]}, \quad \quad a_{m} \neq 0, a_{m} \in J,
$$

relative to a chosen sequence of twisted powers for $x$. Since $H$ is graded we may also write

$$
a u=\sum a b_{i} u_{i} \quad b_{i} \neq 0, b_{i} \in J,
$$

where the $u_{i}$ are nonzero homogeneous divided elements of different degrees. Comparing (1.9) and (1.10) we see that for each $i$ there is a unique $m$ such that

$$
a b_{i} u_{i}=c_{m} a_{m} x^{[m]},
$$

where $c_{m} \in J$ is a unit. From Proposition 1.1(b) it follows that $a$ divides $a_{m}$. Dividing through (1.9) by $a$, we see that $u \in J[x, h(x), t(x)]$. The proposition now follows from the preceding lemma.

CoRollary 1.4. Under the assumptions of Proposition 1.3, $J[x, h(x), t(x)] \otimes J[x, h(x), t(x)]$ is a direct summand of $H \otimes H$.

Let $H$ be a graded anticommutative $J$-algebra with the property that $h(x)=2$ if $x$ has odd degree. Then $H$ is said to possess a system of divided powers [3, Exposé 7] if to each element $x$ of positive even degree there corresponds a sequence of elements $\gamma_{m}(x)$ $\in H(m=0,1,2, \cdots)$ such that:

$$
\begin{aligned}
& \gamma_{0}(x)=1, \quad \gamma_{1}(x)=x, \quad \operatorname{deg} \gamma_{m}(x)=m \cdot \operatorname{deg} x, \\
& \gamma_{r}(x) \gamma_{s}(x)=(r, s) \gamma_{m}(x), \\
& \gamma_{m}(x+y)=\sum_{r+s=m} \gamma_{r}(x) \gamma_{s}(y), \\
& \gamma_{m}(x y)= \begin{cases}0 & \text { if } m \geqq 2 \text { and } x \text { and } y \text { have odd degrees, } \\
x^{m} \gamma_{m}(y) & \text { if } x \text { has even and } y \text { positive even degree. }\end{cases}
\end{aligned}
$$

By induction one proves readily

$$
m ! \gamma_{m}(x)=x^{m} .
$$


(Compare (1.2) $)^{\prime}$ and note that if the characteristic of $J$ is a prime $p$ then (1.15) also implies that $h(x) \leqq p$.) Now assume $H$ is a free module. If $x$ is divided we may choose a sequence of twisted powers for $x$. Since each $x^{[m]}$ is a divided element, in view of Proposition 1.1(b) it follows from (1.15) and (1.2) that $m$ ! divides $g\left(x^{m}\right)$ and, consequently, that $(r, s)$ divides $t_{r, s}(x)$.

2. Hopf algebras with divided powers. A Hopf algebra consists of a pair $(H, \Delta)$, where $H$ is a graded, anticommutative, and associative $J$-algebra with unit 1 (we assume $H^{i}=0$ if $i<0, H^{0}=J \cdot 1$ ), and $\Delta: H \rightarrow H \otimes H$ is an algebraic homomorphism (called the coproduct) such that

$$
\Delta(x)-\Delta^{\prime}(x) \in H^{+} \otimes H^{+}, \quad x \in H, H^{+}=\sum_{i>0} H^{i},
$$

where

$$
\Delta^{\prime}(x)=\left\{\begin{array}{lr}
a(1 \otimes 1) & \text { if } x=a \cdot 1, a \in J, \\
x \otimes 1+1 \otimes x & \text { if } x \in H^{+} .
\end{array}\right.
$$

An element $x$ is said to be primitive if $\Delta(x)=\Delta^{\prime}(x)$. The subset of primitive elements is a submodule which we denote by $\pi_{0}$. Let $\pi$ denote the subalgebra generated by the primitive elements; then $H$ is called a primitive Hopf algebra if $H=|\pi|$. We shall make use of the following theorems:

TheOREM A. Let $(H, \Delta)$ be a Hopf algebra of finite type over a field $K$ of characteristic $p$ such that if $p$ is prime then $u^{p}=0$ for all $u \in H$. If $\left\{1, x_{1}, x_{2}, \cdots, x_{i}, \cdots\right\}$ is a minimal sequence of generators then algebraically

$$
H \cong \underset{i}{\otimes} K\left[x_{i}\right] /\left(x^{h_{i}}\right), \quad \quad h_{i}=h\left(x_{i}\right)
$$

where

$$
h_{i}= \begin{cases}2 & \text { if } x_{i} \text { is not in the center of } H, \\ \infty & \text { if } x_{i} \text { is in the center of } B \text { and } p=0, \\ p & \text { if } x_{i} \text { is in the center of } H \text { and } p \neq 0 .\end{cases}
$$

THEOREM B. Under the preceding assumptions, if each $x_{i}$ is primitive then the sequence $\left\{1, x_{1}, x_{2}, \cdots, x_{i}, \cdots\right\}$ is a basis for the primitive subspace $\pi_{0}$.

TheOREM C. Let $(H, \Delta)$ be a torsion-free Hopf algebra of finite type over a principal ideal domain $J$ of characteristic $p$. If $\left\{x_{1}\right.$, 
$\left.x_{2}, \cdots, x_{i} \cdots\right\}$ is a special sequence of pseudogenerators such that each $x_{i}$ is primitive and of binomial type and the submodule spanned by the $x_{i}$ is a direct summand then

$$
H \cong \underset{i}{\otimes} J\left\langle x_{i}\right\rangle /\left(x^{h_{i}}\right),
$$

where each height $h_{i}=h\left(x_{i}\right)$ satisfies (2.1).

REMARKs. Theorem A is a portion of Borel's Theorem cited above. The assumption in [1] that the field $K$ be perfect is superfluous when $p=0$; if $p \neq 0$ it may be replaced by the above condition that $u^{p}=0$ for all $u \in H$. The proof of Theorem $\mathrm{A}$ in the latter case is then essentially the same as for the case $p=0$. Theorem $B$ is proved in [4] (Corollaries 2.5 and 2.6). Theorem $\mathrm{C}$ is a restatement of Theorem 7.13 in [5]. Note that as stated in [5] it is required that the submodule spanned by the set

$$
\begin{array}{ll}
X=\left\{1, x_{1}, x_{2}, \cdots, x_{i}, \cdots\right\} & \text { if } p=0, \\
X^{\prime}=\left\{x_{i}{ }^{[p]} ; 0 \leqq p^{k}<h_{i}, i=1,2, \cdots\right\} & \text { if } p \neq 0,
\end{array}
$$

be a direct summand of $H$. In the latter case, however, since each $x_{i}$ is of binomial type, it follows that $h_{i} \leqq p$ (see remark after $\left.(1.2)^{\prime}\right)$ and hence $X^{\prime}=X$. Note also that the $x_{i}$ are divided elements.

Proposition 2.1. Let $(H, \Delta)$ be a torsion-free Hopf algebra of finite type over a principal ideal domain $J$. If $x \in H^{i}(i>0)$ is a primitive divided element then it is of pseudobinomial type.

Proof. Choose a sequence of twisted powers for $x$. If $h(x)=2$ the proposition is trivial. Suppose $h(x)>2$; then $x$ is in the center of $H$. Since $x$ is primitive we may write, using the binomial theorem and (1.2),

$$
g\left(x^{m}\right) \Delta\left(x^{[m]}\right)=\sum_{r+8=m}(r, s) g\left(x^{r}\right) g\left(x^{s}\right) x^{[r]} \otimes x^{[s]}, \quad 1 \leqq m<h(x) .
$$

In view of Corollary 1.4, $g\left(x^{m}\right)$ divides each coefficient in the summation. From (1.6) it then follows that $t_{r, 8}(x)$ divides $(r, s)$.

Let $(H, \Delta)$ be a torsion-free Hopf algebra over a principal ideal domain $J$. Let $K$ be the quotient field of $J$ with grading $K^{0}=K$ and $K^{i}=0$ if $i>0$. On the graded $K$-algebra $K \otimes H$ we define a coproduct $\Delta_{K}$ by

$$
\Delta_{K}(a \otimes u)=a \otimes \Delta(u), \quad a \in K, u \in H .
$$

One checks readily that $\left(K \otimes H, \Delta_{K}\right)$ is a Hopf algebra over $K$. We note: 
(i) $K \otimes \pi_{0}$ is its primitive subspace.

(ii) $K \otimes \pi$ is its primitive subalgebra.

(iii) $K \otimes H$ is primitive if and only if $H$ is primitive.

Since $H$ is torsion-free it follows from (2.2) that $a \otimes u$ is primitive if and only if $u$ is primitive and hence (i) is clear. (ii) is obvious in view of (i). To prove (iii) note that $K \otimes|\pi|=K \otimes \pi$. Thus if $H$ is primitive we have

$$
K \otimes H=K \otimes|\pi|=K \otimes \pi .
$$

Conversely, if $K \otimes H$ is primitive we have

$$
K \otimes \pi=K \otimes H \supset K \otimes|\pi| K \otimes \pi,
$$

and hence $H=|\pi|$. The main theorem is as follows:

TheOREM 2.2. Let $(H, \Delta)$ be a torsion-free primitive Hopf algebra of finite type over a principal ideal domain $J$. Then $H$ has a system of divided powers if and only if

$$
H \cong \underset{i}{\otimes J}\left\langle x_{i}\right\rangle /\left(x_{i}^{h_{i}}\right),
$$

where the $x_{i}$ are primitive homogeneous (divided) elements and the $h_{i}=h\left(x_{i}\right)$ satisfy (2.1).

Proof. Suppose $H$ is isomorphic to such a tensor product. If only one pseudogenerator $x=x_{1}$ occurs, define $\gamma_{m}(x)=x^{[m]}$; if more than one occurs then the existence of a system of divided powers follows from Theorem 2 in [2].

Conversely, suppose $H$ possesses a system of divided powers. We first show that the primitive submodule $\pi_{0}$ is a direct summand. Let $u \in H$ and $a \in J, a \neq 0$, be such that $a u \in \pi_{0}$. Then

$$
a\left[\Delta(u)-\Delta^{\prime}(u)\right]=\Delta(a u)-\Delta^{\prime}(a u)=0 .
$$

Since $H$ is torsion-free, it follows that $\Delta(u)-\Delta^{\prime}(u)=0$, that is, $u \in \pi_{0}$. Thus $\pi_{0}$ is rationally closed and hence a direct summand.

Choose a basis $X=\left\{1, x_{1}, x_{2}, \cdots, x_{i}, \cdots\right\}$ for $\pi_{0}$ with each $x_{i}$ homogeneous. We assert that the divided elements $x_{i}$ are of binomial type. Choose a sequence of twisted powers for $x_{i}$. By the remark following (1.15) we know that if $r+s<h_{i}$ then $(r, s)$ divides $t_{r, 8}\left(x_{i}\right)$. The converse is a consequence of Proposition 2.1, and the assertion is proved. It remains only to show that the sequence $X$ is a special system of pseudogenerators in order to apply Theorem $\mathrm{C}$ and complete the proof of Theorem 2.2.

Let $K$ be the quotient field of $J$ and consider the Hopf algebra 
( $\left.K \otimes H, \Delta_{K}\right)$ defined above. As a consequence of (i), (ii), and (iii) we know that it is primitive and that the sequence

$$
\bar{X}=\left\{\overline{1}, \bar{x}_{1}, \bar{x}_{2}, \cdots, \bar{x}_{i}, \cdots\right\} \quad \overline{1}=1 \otimes 1, \bar{x}_{i}=1 \otimes x_{i},
$$

is a basis for $K \otimes \pi_{0}$ and a system of generators for $K \otimes \pi$. We shall prove that it is minimal. Assume that the corresponding sequence of degrees of the $x_{i}$ is nondecreasing. We proceed by induction on $i$. Let $i>1$ and assume

$$
\bar{x}_{j} \notin P\left(\bar{x}_{1}, \bar{x}_{2}, \cdots, \bar{x}_{j-1}\right) \quad \text { for } 1<j \leqq i .
$$

Note that (2.3) is trivial if $i=1$. We shall show that (2.3) holds if we replace $i$ by $i+1$. Suppose not; then

$$
\bar{x}_{i+1} \in P\left(\bar{x}_{1}, \bar{x}_{2}, \cdots, \bar{x}_{i}\right) .
$$

Now the subalgebra $P\left(\bar{x}_{1}, \bar{x}_{2}, \cdots, \bar{x}_{i}\right)$ is clearly a Hopf algebra under (the restriction of) $\Delta_{K}$ with each $x_{j}$ primitive and of binomial type. Moreover, by the inductive assumption, the sequence $\left\{\overline{1}, \bar{x}_{1}\right.$, $\left.\bar{x}_{2}, \cdots, \bar{x}_{i}\right\}$ is a minimal system of generators. Applying Theo-

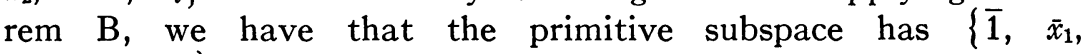
$\left.\bar{x}_{2}, \cdots, \bar{x}_{i}\right\}$ as basis. Since $\bar{x}_{i+1}$ is primitive it follows from (2.4) that

$$
\bar{x}_{i+1}=a_{1} \bar{x}_{1}+a_{2} \bar{x}_{2}+\cdots+a_{i} \bar{x}_{i}, \quad a_{j} \in K .
$$

But this contradicts the fact that $\bar{X}$ is a basis for $K \otimes \pi_{0}$. This completes the induction and the proof of minimality.

Consider the commutative diagram

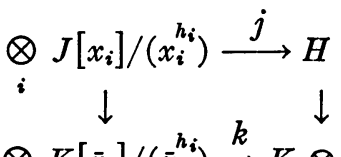

$$
\begin{aligned}
& \otimes K\left[\bar{x}_{i}\right] /\left(\bar{x}_{i}^{h_{i}}\right) \stackrel{k}{\rightarrow} K \otimes H, \quad h_{i}=h\left(x_{i}\right)=h\left(\bar{x}_{i}\right),
\end{aligned}
$$

where the vertical maps are the algebra monomorphisms induced by the injections $u \rightarrow 1 \otimes u$, and the horizontal maps are induced by inclusions. By Theorem A, $k$ is an algebra isomorphism; hence $j$ is a monomorphism and the sequence $X$ is a special system of pseudogenerators for $H$. Theorem 2.2 thus follows from Theorem $\mathrm{C}$.

We have the following application to $H$-spaces:

TheOREM 2.3. Let $T$ be an $H$-space with homotopy-associative and homotopy-commutative multiplication whose integral cohomology $H^{*}(T, Z)$ is of finite type and torsion-free. Then $H^{*}(T, Z)$ has a system of divided powers if and only if 


$$
H^{*}(T, Z) \cong \otimes_{i} Z\left\langle x_{i}\right\rangle /\left(x_{i}^{h_{i}}\right),
$$

where the $x_{i}$ are primitive homogeneous (divided) elements and $h_{i}=h\left(x_{i}\right)$ is 2 or $\infty$ according to whether $x_{i}$ has odd or even degree respectively.

Proof. Let $Q$ be the rational field; then by Theorem 2.10 in [4], $H^{*}(T, Q)$ is a primitive Hopf algebra. Since we may identify

$$
H^{*}(T, Q)=Q \otimes H^{*}(T, Z)
$$

and the coproducts $\Delta_{Q}$ and $\Delta$ in $H^{*}(T, Q)$ and $H^{*}(T, Z)$, respectively, are related by

$$
\Delta_{Q}(a \otimes u)=a \otimes \Delta(u), \quad a \in Q, u \in H^{*}(T, Z),
$$

it follows from (iii) that $H^{*}(T, Z)$ is primitive. Theorem 2.3 thus follows from Theorem 2.2.

For the sake of completeness we state the following theorem which follows from Theorem 2.2 (above) and Theorems 6.4 and 6.5 in [5].

THEOREM 2.4. Under the assumptions of Theorem 2.2, $H$ has a system of divided powers if and only if for the dual Hopf algebra $H^{*}$ we have

$$
H^{*} \cong \underset{i}{\otimes}\left[u_{i}\right] /\left(u_{i}^{h_{i}}\right)
$$

where the $u_{i}$ are primitive homogeneous elements in 1-1 dual correspondence with the $x_{i}$ and $h_{i}=h\left(u_{i}\right)=h\left(x_{i}\right)$.

3. An example. R. Bott proved

$$
H_{*}\left(\Omega G_{2}, Z\right)=Z[u, v, w] /\left\{u^{2}-2 v\right\}
$$

where $\Omega G_{2}$ is the loop space over the exceptional Lie group $G_{2}$ (see $[2$, p. 60$])$. It is readliy seen that the formulas

$$
\begin{aligned}
u^{[2 m]} & =v^{m}, & g\left(u^{2 m}\right) & =2^{m}, \\
u^{[2 m+1]} & =u v^{m}, & g\left(u^{2 m+1}\right) & =2^{m}
\end{aligned}
$$

define a sequence of twisted powers for $u$. In particular, note that $u^{3}$ is not divisible by 3 ! so that (1.15) fails and $H_{*}\left(\Omega G_{2}, Z\right)$ has no system of divided powers. In terms of twisted powers we may write

$$
H_{*}\left(\Omega G_{2}, Z\right) \cong Z[u, h(u), t(u)] \otimes Z[w]
$$

where the height $h(u)=\infty$ and the type $t(u)$ is defined by (1.6) and (3.1). Also, using Theorem 6.4 in [5] we may write 


$$
H^{*}\left(\Omega G_{2}, Z\right) \cong Z[x, h(x), t(x)] \otimes Z\langle y\rangle
$$

where $h(x)=\infty$ and $t(x)$ is defined by the greatest divisors $g\left(x^{n}\right)$ $=n ! / g\left(u^{n}\right)$. In particular, $g\left(x^{2}\right)=1$ so that (1.15) fails for $x^{2}$ and $H^{*}\left(\Omega G_{2}, Z\right)$ has no system of divided powers.

\section{BIBLIOGRAPHY}

1. A. Borel, Sur la cohomologie des espaces fibrés principaux et des espaces homogènes de groupes de Lie compacts, Ann. of Math. vol. 57 (1953) pp. 115-207.

2. R. Bott, The loop space on a Lie group, Michigan Math. J. vol. 5 (1958) pp. 35-67.

3. H. Cartan, Séminaire de Topologie de l'Ecole Normale Supérieure, Paris, 19541955, Notes polycopiées.

4. E. Halpern, On the structure of hyperalgebras. Class 1 Hopf algebras, Portugal. Math. vol. 17 (1958) p. 127-147. 1958.

5. —-, Twisted polynomial hyperalgebras, Memoir Amer. Math. Soc., no. 29,

UNIVERSITY OF MrChigaN

\section{ANOTHER CUTPOINT THEOREM FOR PLANE CONTINUA}

\section{F. BURTON JONES ${ }^{1}$}

If the subcontinuum $M$ of a topological 2-sphere $S$ does not separate $S$ and is locally connected, then each pair of points of $M$, which are not separated in $M$ by a point of $M$, belongs to the closure of a connected domain (of $S$ ) lying in $M$. This is true because each such pair of points belongs to a simple closed curve $J$ lying in $M$ and one of the complementary domains of $J$ is a subset of $M$. However, without local connectedness such a simple closed curve may fail to exist. In fact, the proposition would then be false because (to take an extreme case) of the existence of indecomposable subcontinua of $S$ which fail to separate $S$. While no point of an indecomposable continuum separates it, every point of it cuts it. Recently I showed [1] that this stronger form of separation (or rather the lack of it) is sufficient to restore the validity of the above proposition in the absence of local connectedness if a certain restriction were placed upon

Presented to the Society, August 29, 1958; received by the editors September 15, 1959.

${ }_{1}$ This research was supported (in part) by the United States Air Force through the Air Force Office of Scientific Research of the Air Research and Development Command, under contract No. AF18(600)-1109. Reproduction in whole or in part is permitted for any purpose of the United States Government. 